\title{
ON THE DEFORMATION OF COMMUTATIVE ALGEBRAS
}

\author{
BY \\ DAVID W. KNUDSON( $\left.{ }^{1}\right)$
}

Introduction. Given a field $k$ and a $k$-algebra $A$, Gerstenhaber has introduced the concept of a deformation of $A[8]$ which is a $k[[t]]$-algebra structure on the module $A[[t]]$. One of the main problems of the theory of deformations is to determine those algebras for which the only deformation (modulo an equivalence relation) is the usual $k[[t]]$-algebra structure on $A[[t]]$. Such algebras are said to be rigid. If $A$ is a commutative $k$-algebra, we may restrict our attention to those deformations of $A$ which are commutative. This leads us to consider the commutative cohomology groups of $A, H^{n}(A, M)^{s}$, defined by Harrison [11]. The principal purpose of this paper is to investigate the following conditions on a commutative algebra $A$ over a field $k$ :

(1) $H^{2}(A, M)^{s}=0$ for all $A$-modules $M$.

(2) $H^{2}(A, A)^{s}=0$.

(3) $A$ is a rigid $k$-algebra in the commutative deformation theory.

(4) The module of differentials of $A$ over $k$ is a projective $A$-module.

(5) $A$ is a finite product of (not necessarily algebraic) separable extension fields of $k$.

We may show that (1) implies (4) [9, Theorem (20.4.9)] and that (2) implies (3) [8, p. 65]. Condition (1) may be expressed by saying that $A$ is a formally smooth $k$-algebra $[9, \S 19.4 .4]$.

When $A$ is a field, Gerstenhaber has shown that (1), (2), and (5) are equivalent and he conjectured that these conditions were equivalent to (3). We show that (1), (2), (3), and (5) are equivalent even for $A$ semisimple (Theorem 6.5). Moreover, if $A$ is artinian, then (1) and (5) are equivalent (Proposition 5.9). In another direction, if $A$ is a geometric complete intersection, then (1), (2), and (3) are equivalent (Theorem 6.8a). If in addition $A$ is an integral domain with a separable quotient field, then (1), (2), (3), and (4) are equivalent (Theorem 6.8c).

In the final section, we investigate the above conditions for the group algebra $B[G]$ of an abelian group $G$ which is a direct sum of cyclic groups with $B$ a commutative algebra over a field $k$ of positive characteristic $p$. In addition, we consider the following condition on $G$ and $k$ :

(6) $G$ has no element of order $p=\operatorname{char}(k)$.

Received by the editors May 10, 1967 and, in revised form, July 31, 1968.

( ${ }^{1}$ This research was supported by an NSF Cooperative Graduate Fellowship at Northwestern University and by an ONR Research Associateship at Cornell University. 
If $B$ is a noetherian formally smooth $k$-algebra, we show that (1) and (6) are equivalent for the algebra $A=B[G]$ (Theorem 7.2). If in addition $B$ is a geometric complete intersection or a semisimple $k$-algebra, then (1), (2), (3), and (6) are equivalent (Corollary 7.3).

The results on rigidity depend on the vanishing of the third commutative cohomology groups of the algebra in question. We show that if $A$ is a geometric complete intersection or if $A$ is semisimple, then $H^{3}(A, M)^{s}=0$ for all $A$-modules $M$ (Corollary 6.4). The essential tools for the computation of these groups are an exact sequence of Harrison [11, Theorem 2] and a representation of the symmetric cochain complex by means of a chain complex. In $\S 2$, we discuss the relationship of the groups $\left(\operatorname{Ext}_{A}^{n}\left(\Omega_{A \mid k}, M\right)\right)_{n \geqq 0}$ and $\left(H^{m}(A, M)^{s}\right)_{m \geqq 1}$ where $\Omega_{A / k}$ denotes the module of differentials of $A$ over $k$.

This paper is the author's doctoral dissertation written under the direction of Professor Daniel Zelinsky at Northwestern University. I would like to express my appreciation to Professor Zelinsky for his encouragement and advice during the preparation of this thesis. I would also like to thank the referee for many valuable suggestions and comments.

Notation. All rings will be assumed to have an identity and a ring homomorphism wili preserve the identity. The expression " $A$ is a $k$-algebra" will imply that $k$ is a field. Except for $\S 1$, all rings will be commutative.

1. Preliminary remarks. Let $A$ be a $k$-algebra, $M$ an $A$-bimodule, and $C^{n}(A, M)$ the $k$-module of all $n$-linear maps over $k$ of $A$ into $M$. As usual [13], we define the coboundary operator $\delta$ by

$$
\begin{aligned}
\delta_{n} f\left(a_{1}, \ldots, a_{n+1}\right)= & a_{1} f\left(a_{2}, \ldots, a_{n+1}\right) \\
& +\Sigma_{1 \leqq i \leqq n}(-1)^{i} f\left(a_{1}, \ldots, a_{i} a_{i+1}, \ldots, a_{n+1}\right) \\
& +(-1)^{n+1} f\left(a_{1}, \ldots, a_{n}\right) a_{n+1}
\end{aligned}
$$

where $f \in C^{n}(A, M)$. The $n$th cohomology group of this complex is denoted by $H^{n}(A, M)=Z^{n}(A, M) / B^{n}(A, M)$ and the elements of $Z^{n}(A, M)=\operatorname{ker}\left(\delta_{n}\right)$ and $B^{n}(A, M)=\operatorname{im}\left(\delta_{n-1}\right)$ are called $n$-cocycles and $n$-coboundaries respectively.

Let $A[[t]]$ denote the formal power series ring in one variable over $A$. A deformation of the $k$-algebra $A[8]$ is an associative $k[[t]]$-bilinear map $f_{t}$ on $A[[t]]$ which is expressible in the form

$$
f_{t}(a, b)=a b+t f_{1}(a, b)+t^{2} f_{2}(a, b)+\cdots
$$

where " $a b$ " denotes the usual product in $A[[t]]$ and where each $f_{i}$ is a $k$-bilinear map on $A$ extended in the natural manner to a $k[[t]]$-bilinear map on $A[[t]]$.

The associativity condition on $f_{t}$ is equivalent to the system of equations

$$
\Sigma_{0<p<n} f_{p}\left(f_{n-p}(a, b), c\right)-f_{p}\left(a, f_{n-p}(b, c)\right)=\delta f_{n}(a, b, c)
$$

for all $a, b, c \in A$ and each $n=0,1,2, \ldots$ Note that $f_{1} \in Z^{2}(A, A)$. One may show 
that if $H^{3}(A, A)=0$, then every element of $Z^{2}(A, A)$ determines a deformation of $A$ $[8$, p. 64].

Let $f_{t}$ and $g_{t}$ be deformations of $A$. We say that $f_{t}$ is equivalent to $g_{t}$ if there is a $k[[t]]$-linear automorphism $\mu_{t}$ of $A[[t]]$ of the form

$$
\mu_{t}(a)=a+t \mu_{1}(a)+t^{2} \mu_{2}(a)+\cdots
$$

where each $\mu_{i}$ is a $k$-linear map on $A$ extended in the natural manner to a $k[[t]]-$ linear map on $A[[t]]$ such that $\mu_{t}\left(g_{t}(a, b)\right)=f_{t}\left(\mu_{t}(a), \mu_{t}(b)\right)$ for all $a, b \in A[[t]]$. We may easily check that $g_{1}=f_{1}+\delta \mu_{1}$ in this case. A deformation $f_{t}$ of $A$ is said to be trivial if $f_{t}$ is equivalent to the deformation $g_{t}$ of $A$ defined $g_{t}(a, b)=a b$. Thus if the cocycle $f_{1}$ of the deformation $f_{t}$ is not a coboundary, it follows that $f_{t}$ is a nontrivial deformation. If every deformation of $A$ is trivial, we say that $A$ is a rigid $k$-algebra. Gerstenhaber proved that if $H^{2}(A, A)=0$, then $A$ is a rigid $k$-algebra [8, p. 65]. In general, the converse is not known. We refer the reader to [8] for a detailed discussion of the deformation of an algebra.

If the $k$-algebra $A$ is commutative, it is natural to consider only those deformations $f_{t}$ of $A$ which define a commutative structure on $A[[t]] . f_{t}$ will be a commutative deformation of $A$ if and only if $f_{i}(a, b)=f_{i}(b, a)$ for all $a, b \in A$ and each $i=1,2, \ldots$ Thus we are led to the commutative cohomology theory of Harrison [11]. Since we shall be concerned with this cohomology theory only through dimension 3 , we shall limit our discussion to these groups.

If $A$ is a commutative $k$-algebra and $M$ an $A$-module, Harrison defines a certain subcomplex $\left(C^{n}(A, M)^{s}\right)_{n \geqq 1}$ of Hochschild's complex. We shall call the elements of these groups symmetric n-cochains. $C^{n}(A, M)^{s}$ is the set of all $F \in C^{n}(A, M)$ such that $F(a, b)=F(b, a)$ for all $a, b \in A$ if $n=2$ and such that $F(a, b, c)-F(b, a, c)$ $+F(b, c, a)=0$ for all $a, b, c \in A$ if $n=3$. We set $C^{1}(A, M)^{s}=C^{1}(A, M)$ and let

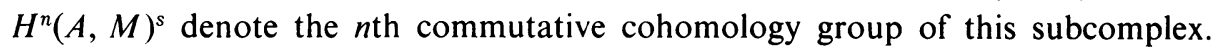
The reader should note that $H^{1}(A, M)=H^{1}(A, M)^{\mathrm{s}}=\operatorname{Der}_{k}(A, M)$, the $A$-module of $k$-derivations of $A$ into $M$, and that $H^{2}(A, M)^{s}$ is a submodule of $H^{2}(A, M)$. All of the above definitions and remarks apply to the commutative deformation theory simply by replacing the Hochschild cohomology theory by the Harrison commutative cohomology theory.

Definition 1.1. Let $A$ be a commutative $k$-algebra and let $1 \leqq n \leqq 4$. Set $A^{n+1}$ $=A \otimes_{k} \cdots \bigotimes_{k} A$ ( $n+1$ factors) and consider $A^{n+1}$ as an $A$-module by multiplication on the first factor. Let $I_{n}$ be the submodule of $A^{n+1}$ generated by elements of the form

$$
\begin{aligned}
x_{0} \otimes \cdots \otimes x_{n} & +\Sigma_{1<i<n}(-1)^{i-1} x_{0} \otimes x_{2} \otimes \cdots \otimes x_{i} \otimes x_{1} \otimes x_{i+1} \otimes \cdots \otimes x_{n} \\
& +(-1)^{n-1} x_{0} \otimes x_{2} \otimes \cdots \otimes x_{n} \otimes x_{1}
\end{aligned}
$$

for $n=2,3$, and 4 and set $I_{1}=0$. We define the module of symmetric $n$-chains of $A$ over $k$ to be $C_{n}(A / k)^{s}=A^{n+1} / I_{n}$. 
By direct computation, we may check that the mapping $d_{n}: A^{n+1} \rightarrow A^{n}$ defined by

$$
\begin{aligned}
d_{n}\left(x_{0} \otimes \cdots \otimes x_{n}\right)= & x_{0} x_{1} \otimes \cdots \otimes x_{n}-x_{0} \otimes x_{1} x_{2} \otimes \cdots \otimes x_{n}+\cdots \\
& +(-1)^{n} x_{n} x_{0} \otimes \cdots \otimes x_{n-1}
\end{aligned}
$$

maps $I_{n}$ into $I_{n-1}$ and that $d_{n}$ defines a boundary operator on the complex $C_{*}(A \mid k)^{s}$. We shall denote the homology groups of this complex by $H_{n}(A / k)^{s}$.

We may easily check that $C^{*}(A, M)^{s} \cong \operatorname{Hom}_{A}\left(C_{*}(A / k)^{s}, M\right)$ as cochain complexes through dimension 3 . Thus if we have available a universal coefficient theorem, this representation of the symmetric cochain complex gives us the possibility of applying direct limit arguments since homology commutes with exact functors of complexes.

We recall that if $A \cong \prod_{1 \leqq i \leqq m} A_{i}$ is a product of commutative rings and if $M$ is an $A$-module, then $M \cong \prod_{1 \leqq i \leqq m} M_{i}$ where $M_{i}=e_{i} M$ with $e_{i}=(0, \ldots, 1,0, \ldots, 0)$, the 1 being the $i$ th position. In addition, $M_{i} \cong A_{i} \otimes_{A} M$ [16, Chapter IX, Proposition 9.2]. Whenever $H^{n}(A, M)^{s}$ is contained in $H^{n}(A, M)$, we may easily show that $H^{n}(A, M)^{s} \cong \prod_{1 \leqq i \leqq m} H^{n}\left(A_{i}, M_{i}\right)^{s}$ by using the corresponding result for the Hochschild cohomology groups [13, Theorem 4]. Unfortunately, it is not known whether or not $H^{3}(A, M)^{s}$ is contained in $H^{3}(A, M)$ if char $(k)=2$. However, the above isomorphism will be true in this case. Michael Barr has communicated to me a proof due to Michel André which establishes this isomorphism for dimensions 1, 2, and 3. (More generally, the proof establishes this isomorphism whenever Harrison's cohomology agrees with the cohomology groups defined via cotriples.)

Definition 1.2. Let $L$ be an extension field of $k$. We say that $L$ is a separable extension of $k$ if one of the following equivalent conditions is satisfied:

(1) Every finitely generated subfield of $L$ is separably generated over $k$.

(2) $L$ and $k^{1 / p}$ are linearly disjoint over $k$.

We refer the reader to [5] for details. Recall also that Gerstenhaber proved that these conditions are equivalent to the following conditions [8, Theorem 3]:

(3) $H^{2}(L, L)^{s}=0$.

(4) $H^{2}(L, M)^{s}=0$ for all $L$-modules $M$.

2. The module of differentials. For the remainder of this paper, all rings will be assumed to be commutative. Let $z(A)$ be the set of zero-divisors of a ring $A$. We recall that the total quotient ring of $A$ is the localization of $A$ at the multiplicatively closed set $A-z(A)$. If $M$ is an $A$-module, an element $m \in M$ is said to be a torsion element if there exists $a \in A-z(A)$ such that $a m=0$. We say that $M$ is torsion-free if the submodule of torsion elements of $M$ is zero. Note that a submodule of a free module is torsion-free. We shall need the following lemma.

Lemma 2.1. Let $A$ be a (not necessarily commutative) ring and $X$ an A-module. If $\operatorname{Ext}_{A}^{n+1}(X, M)=0$ whenever $M$ is a submodule of a free $A$-module, then the projective dimension of $X$ is $\leqq n$. 
Proof. Let $0 \rightarrow K \rightarrow F \rightarrow X \rightarrow 0$ be an exact sequence of $A$-modules where $F$ is free.

Suppose $n=0$. We obtain an exact sequence

$$
0 \rightarrow \operatorname{Hom}_{A}(X, K) \rightarrow \operatorname{Hom}_{A}(X, F) \rightarrow \operatorname{Hom}_{A}(X, X) \rightarrow \operatorname{Ext}_{A}^{1}(X, K)=0 .
$$

Hence $X$ is projective since the map $F \rightarrow X$ splits.

By induction, suppose that the lemma is true for all $0 \leqq m<n$. It will suffice to show that the projective dimension of $K$ is $\leqq n-1$. Let $M$ be a submodule of a free $A$-module. Then

$$
0=\operatorname{Ext}_{A}^{n}(F, M) \rightarrow \operatorname{Ext}_{A}^{n}(K, M) \rightarrow \operatorname{Ext}_{A}^{n+1}(X, M)=0
$$

is an exact sequence and so the projective dimension of $K$ is $\leqq n-1$ by induction.

Definition 2.2. Let $A$ be a $k$-algebra and $M$ an $A$-module. The module of $k$-differentials of $A$ is an $A$-module $\Omega_{A / k}$ and a $k$-derivation $d: A \rightarrow \Omega_{A / k}$ which is universal with respect to $k$-derivations of $A$ into $A$-modules. Hence we have a natural isomorphism

$$
\operatorname{Hom}_{A}\left(\Omega_{A / k}, M\right) \cong \operatorname{Der}_{k}(A, M)
$$

where $M$ is an $A$-module.

In the terminology of [10, Chapter 2], $\left(\operatorname{Ext}_{A}^{n}\left(\Omega_{A / k}, \cdot\right)\right)_{n \geqq 0}$ is a universal connected $\delta$-functor. Since $\left(H^{m}(A, \cdot)\right)_{m \geqq 1}$ is a connected $\delta$-functor [11, Theorem 1], there is a unique morphism $\left(\mu_{n}\right)_{n \geqq 0}$ of $\delta$-functors where

$$
\mu_{n}: \operatorname{Ext}_{A}^{n}\left(\Omega_{A / k}, \cdot\right) \rightarrow H^{n+1}(A, \cdot)^{s}
$$

and $\mu_{0}$ is the isomorphism of (1).

Proposition 2.3. Let $A$ be a k-algebra and $M$ an A-module. The map

$$
\mu_{1}: \operatorname{Ext}_{A}^{1}\left(\Omega_{A / k}, M\right) \rightarrow H^{2}(A, M)^{s}
$$

is a monomorphism.

Proof. Let $0 \rightarrow M \rightarrow I \rightarrow I / M \rightarrow 0$ be an exact sequence where $I$ is an injective $A$-module. This sequence induces the following commutative diagram with exact rows where $\Omega=\Omega_{A / k}$.

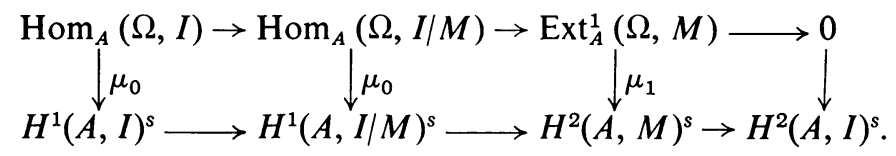

Since $\mu_{0}$ is an isomorphism, $\mu_{1}$ is a monomorphism by the four lemma [16, p. 14].

In general, $\mu_{1}$ is not an epimorphism. If $A$ is any field, then $\operatorname{Ext}_{A}^{1}\left(\Omega_{A / k}, M\right)=0$. But if $A$ is not a separable extension of $k, H^{2}(A, M)^{s} \neq 0$ [8, Theorem 3].

Corollary 2.4. Let $A$ be a k-algebra. If $H^{2}(A, M)^{s}=0$ whenever $M$ is a submodule of a free $A$-module, then $\Omega_{A / k}$ is a projective $A$-module. 
Proof. Apply Lemma 2.1 and Proposition 2.3.

We may show that the zero ideal of a ring $A$ is the intersection of a finite number of prime ideals if and only if the total quotient ring of $A$ is a finite product of fields [2, p. 117].

Proposition 2.5. Let $A$ be a k-algebra such that the total quotient ring of $A$ is a finite product of separable extension fields of $k$. If $M$ is a torsion-free A-module, then

$$
\mu_{n}: \operatorname{Ext}_{A}^{n}\left(\Omega_{A / k}, M\right) \rightarrow H^{n+1}(A, M)^{s}
$$

is an isomorphism for $n=1$ and a monomorphism for $n=2$.

Proof. Let $S^{-1} A$ be the total quotient ring of $A$ and set $K=S^{-1} A / A$. The sequence $0 \rightarrow M \rightarrow S^{-1} M \rightarrow K \otimes_{A} M \rightarrow 0$ is exact since $M$ is torsion-free. We claim that $S^{-1} M$ is an injective $A$-module. Since a product of injective modules is an injective module, we may assume that $S^{-1} A$ is a field. But if $X$ is any $A$-module, we have an isomorphism [1, $\$ 4$, Proposition 1]

$$
\operatorname{Hom}_{S^{-1} A}\left(S^{-1} X, S^{-1} M\right) \cong \operatorname{Hom}_{A}\left(X, S^{-1} M\right) .
$$

Hence $\operatorname{Hom}_{A}\left(\cdot, S^{-1} M\right)$ is the composite of two exact functors and so $S^{-1} M$ is an injective $A$-module.

By [11, Theorem 16], the map $A \rightarrow S^{-1} A$ induces an isomorphism $H^{2}\left(A, S^{-1} M\right)^{s}$ $\cong H^{2}\left(S^{-1} A, S^{-1} M\right)^{s}$. Hence using the hypothesis on $S^{-1} A$ and the remark following Definition 1.1, we have that $H^{2}\left(A, S^{-1} M\right)^{s}=0$ since the 2nd cohomology group of a separable extension is always zero [8, Theorem 3]. Thus we obtain the following commutative diagrams with exact rows where we set $\Omega=\Omega_{A / k}$.

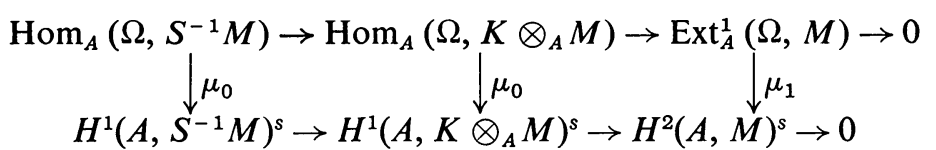

$$
\begin{aligned}
& 0 \rightarrow \operatorname{Ext}_{A}^{1}\left(\Omega, K \otimes_{A} M\right) \rightarrow \operatorname{Ext}_{A}^{2}(\Omega, M) \rightarrow 0 \\
& 0 \rightarrow H^{2}\left(A, K \otimes_{A} M\right)^{s} \rightarrow H^{3}(A, M)^{s} .
\end{aligned}
$$

Since $\mu_{0}$ is always an isomorphism, $\mu_{1}$ is an isomorphism in the first diagram. In the second diagram, $\mu_{1}$ is a monomorphism by Proposition 2.3. Hence $\mu_{2}$ is a monomorphism.

COROLlary 2.6. Let $A$ be a k-algebra which satisfies the hypothesis of Proposition 2.5 .

(a) If $H^{3}(A, M)^{s}=0$ whenever $M$ is a submodule of a free $A$-module, then the projective dimension of $\Omega_{A / k}$ is $\leqq 1$.

(b) If $\Omega_{A / k}$ is a projective $A$-module, then $A$ is a rigid $k$-algebra in the commutative deformation theory. 
Proof. Apply Lemma 2.1 and Proposition 2.5 and note that $H^{2}(A, A)^{s}=0$ under the hypothesis of part (b).

3. The cohomology of a localization of an algebra. The following lemma is a rewording of [14, Lemma 4.1$]$.

Lemma 3.1. Let $A$ be a ring and let $K_{*}$ be a chain complex of $A$-modules such that $K_{i}$ is projectice for $m \leqq i \leqq n$. If $Z_{m}\left(K_{*}\right)$ is projectice and if $H_{i}\left(K_{*}\right)$ is projectice for $m \leqq i<n$, then the natural map

$$
H^{i}\left(\operatorname{Hom}_{A}\left(K_{*}, M\right)\right) \rightarrow \operatorname{Hom}_{A}\left(H^{i}\left(K_{*}\right), M\right)
$$

is an isomorphism for $m \leqq i \leqq n$ where $M$ is an A-module.

Lemma 3.2. Let $B$ be a polynomial $k$-algebra, $S$ a multiplicaticely closed subset of $B$, and $A=S^{-1} B$. Then the complex $C_{*}(A \mid k)^{s}$ of Definition 1.1 satisfies the hypotheses on $K_{*}$ in Lemma 3.1 where $m=1$ and $n=3$.

Proof. Let $A$ be any $k$-algebra and consider $C_{n}(A / k)^{s}$. Let $J_{n}$ be the $k$-submodule of $A^{n}=A \otimes_{k} \cdots \otimes_{k} A$ ( $n$ factors) generated by elements of the form $x_{1} \otimes$ $\cdots \otimes x_{n}-x_{2} \otimes x_{1} \otimes x_{3} \otimes \cdots \otimes x_{n}+\cdots+(-1)^{n-1} x_{2} \otimes \cdots \otimes x_{n} \otimes x_{1}$. Clearly $A \otimes_{k} J_{n} \cong I_{n}$ using the notation of Definition 1.1. Since $A$ is flat over $k, C_{n}(A / k)^{s}$ $\cong A \otimes_{k}\left(A^{n} / J_{n}\right)$ and therefore $C_{n}(A / k)^{s}$ is a free $A$-module. Also note that $Z_{1}(A \mid k)^{s}$ $=C_{1}(A \mid k)^{s}=A \otimes_{k} A$. Now $B_{1}(A \mid k)^{s}$ is generated as an $A$-module by elements of the form $a \otimes b-1 \otimes a b+b \otimes a$. Thus $H_{1}(A / k)^{s} \cong \Omega_{A / k}$ [5].

Now assume that $A=S^{-1} B$ with $B$ a polynomial $k$-algebra. Since $\Omega_{B / k}$ is a free $B$-module $[9, \S 20]$, then $H_{1}(A / k)^{s} \cong \Omega_{A / k} \cong S^{-1} \Omega_{B / k}$ is a free $A$-module. Applying Lemma 3.1 to the case $m=1$ and $n=2$, we have that

$$
H^{2}(A, M)^{s} \cong \operatorname{Hom}_{A}\left(H_{2}(A / k)^{s}, M\right) .
$$

By [11, Theorem 15], the 2nd cohomology group of a localization of a polynomial $k$-algebra is always zero. Hence $H_{2}(A / k)^{s}=0$ and so the hypotheses of Lemma 3.1 are satisfied for $m=1$ and $n=3$.

Let $A$ be a $k$-algebra, $I$ an ideal, and $M$ an $A / I$-module. We shall make repeated use of the following exact sequence [11, Theorem 2]:

$$
\begin{aligned}
0 & \rightarrow H^{1}(A / I, M)^{s} \rightarrow H^{1}(A, M)^{s} \rightarrow \operatorname{Hom}_{A}(I, M) \rightarrow H^{2}(A / I, M)^{s} \\
& \rightarrow H^{2}(A, M)^{s} \rightarrow C^{1}(A, I, M) \rightarrow H^{3}(A / I, M)^{s} \rightarrow H^{3}(A, M)^{s}
\end{aligned}
$$

where $C^{1}(A, I, M)$ is a submodule of $\operatorname{Ext}_{A}^{1}(I, M)$.

THeOREM 3.3. Let $B$ be a polynomial $k$-algebra and $S$ a multiplicatively closed subset of $B$. If $M$ is an $S^{-1} B$-module, then $H^{3}\left(S^{-1} B, M\right)^{s}=0$.

Proof. Let $A=S^{-1} B$. By Lemma 3.2, it will suffice to show that $H_{3}(A / k)^{s}=0$.

Recall that if $B$ is any commutative ring and $S$ is a multiplicatively closed subset of $B$, then $S^{-1} B \cong \operatorname{inj} \lim B_{f}$ where the direct limit is over all $f$ in $S$. $B_{f}$ 
denotes the localization $S_{f}^{-1} B$ where $S_{f}$ is the set of all $f^{n}, n \geqq 0$. Since the process of taking a direct limit over a filtered index set is an exact functor, we have that $C_{*}(A / k)^{s} \cong \operatorname{inj} \lim C_{*}\left(B_{f} / k\right)^{s}$ and $H_{n}(A / k)^{s} \cong \operatorname{inj} \lim H_{n}\left(B_{f} / k\right)^{s}$ where $A=S^{-1} B$. Thus it will suffice to show that $H_{3}\left(B_{f} / k\right)^{s}=0$ for any $f \in B$.

Let $Y$ be an indeterminate over $B$. Then we have an isomorphism $B_{f} \cong B[Y] / I_{f}$ where $I_{f}$ is the ideal in $B[Y]$ generated by $f Y-1$. If $N$ is a $B_{f}$-module, using the exact sequence (1) we obtain an exact sequence

$$
C^{1}\left(B[Y], I_{f}, N\right) \rightarrow H^{3}\left(B_{f}, N\right)^{s} \rightarrow H^{3}(B[Y], N)^{s}=0
$$

since the 3rd cohomology group of a polynomial $k$-algebra is always zero. But since $I_{f}$ is principal and $B[Y]$ is an integral domain, $\operatorname{Ext}_{B[Y]}^{1}\left(I_{f}, N\right)=0$. Hence $H^{3}\left(B_{f}, N\right)^{s}=0$ for all $B_{f}$-modules $N$ and so, using Lemma 3.2, we have that $H_{3}\left(B_{f} / k\right)^{s}=0$.

4. The symmetric algebra of a projective module. Let $A$ be a ring and $M$ an $A$-module. The symmetric $A$-algebra of $M$ is an $A$-algebra $S_{A}(M)$ and an $A$-module map $\gamma: M \rightarrow S_{A}(M)$ which is universal with respect to $A$-module maps of $M$ into $A$-algebras.

If $B$ is an $A$-algebra and if $M$ and $M^{\prime}$ are $A$-modules, then $S_{B}\left(B \otimes_{A} M\right) \cong$ $B \otimes_{A} S_{A}(M)$ and $S_{A}\left(M \oplus M^{\prime}\right) \cong S_{A}(M) \otimes_{A} S_{A}\left(M^{\prime}\right)$. If $M$ is a free $A$-module, then $S_{A}(M)$ is a polynomial $A$-algebra with indeterminates in 1-1 correspondence with a basis of $M$ over $A$.

Theorem 4.1. Let $A$ be a $k$-algebra and $M$ a projective $A$-module. Let $n=2$ or 3 . Then $H^{n}\left(S_{A}(M), N\right)^{s}=0$ for all $S_{A}(M)$-modules $N$ if and only if $H^{n}(A, P)^{s}=0$ for all A-modules $P$.

Proof. If $X$ is a free $A$-module, we will let $X^{*}$ denote a $k$-module with the same number of basis elements as $X$. Hence $X \cong A \otimes_{k} X^{*}$. Since $M$ is projective, there are free $A$-modules $M^{\prime}$ and $F$ such that $M \oplus M^{\prime}=F$. We have the isomorphisms

$$
\begin{aligned}
S_{A}(F) & \cong S_{A}\left(A \otimes_{k} F^{*}\right) \cong A \otimes_{k} S_{k}\left(F^{*}\right) \cong S_{A}\left(M \oplus M^{\prime}\right) \\
& \cong S_{A}(M) \otimes_{A} S_{A}\left(M^{\prime}\right) \cong S_{A}(M) \otimes_{k} S_{k}\left(M^{\prime *}\right) .
\end{aligned}
$$

Recall that the cohomology of a polynomial $k$-algebra is zero in dimensions 2 and 3 [11, Theorem 11]. Hence if $N$ is an $S_{A}(F)$-module

$$
H^{n}\left(S_{A}(F), N\right)^{s} \cong H^{n}(A, N)^{s} \cong H^{n}\left(S_{A}(M), N\right)^{s}
$$

since the cohomology group of a tensor product of two $k$-algebras is isomorphic to the product of the cohomology groups of the $k$-algebras for dimensions 1,2 , and 3 [11, Theorem 5].

Assume that $H^{n}\left(S_{A}(M), N\right)^{s}=0$ for all $S_{A}(M)$-modules $N$ and let $P$ be any $A$-module. Consider $P$ as an $S_{A}(M)$-module via the projection $S_{A}(M) \rightarrow A$. But this is the same as considering $P$ as an $S_{A}(M)$-module via $S_{A}(M) \rightarrow S_{A}(F) \rightarrow A$ 
where the first map is induced by the map $M \rightarrow F$. Since $A \rightarrow S_{A}(M) \rightarrow S_{A}(F) \rightarrow A$ is the identity, the above isomorphism (1) shows that $H^{n}(A, P)^{s}=0$.

Conversely, assume that $H^{n}(A, P)^{s}=0$ for all $A$-modules $P$ and let $N$ be an $S_{A}(M)$-module. $M$ is a direct summand of $F$ and so we have a projection map $F \rightarrow M$ which induces $S_{A}(F) \rightarrow S_{A}(M)$ and $S_{A}(M) \rightarrow S_{A}(F) \rightarrow S_{A}(M)$ is the identity. Hence using the isomorphism of (1), we have that $H^{n}\left(S_{A}(M), N\right)^{s}=0$.

5. Formally smooth algebras and rigidity. The following definition is due to Grothendieck [9, §19].

Definition 5.1. Let $A$ be a $k$-algebra. We say that $A$ is a formally smooth $k$-algebra if $H^{2}(A, M)^{s}=0$ for all $A$-modules $M[9, \S 19.4 .4$.1].

Using this terminology, [8, Theorem 3] may be rephrased as: If $A$ is an extension field of $k$, then $A$ is a separable extension of $k$ if and only if $A$ is a formally smooth $k$-algebra.

Proposition 5.2. (a) Let $\left(A_{i}\right)_{1 \leqq i \leqq n}$ be a collection of $k$-algebras. Then $\prod_{1 \leqq i \leqq n} A_{i}$ is a formally smooth $k$-algebra if and only if each $A_{i}$ is a formally smooth $k$-algebra.

(b) Let $\left(A_{i}\right)_{i \in I}$ be a collection of $k$-algebras. If each $A_{i}$ is a formally smooth $k$-algebra, then the tensor product $\bigotimes_{i \in I} A_{i}$ is a formally smooth $k$-algebra.

(c) Let $\left(A_{i}\right)_{i \in I}$ be a collection of k-algebras where $I$ is a filtered index set and set $A=\operatorname{inj} \lim A_{i}$. If $\Omega_{A / k}$ is a projective A-module and if each $A_{i}$ is a formally smooth $k$-algebra, then $A$ is a formally smooth k-algebra.

Proof. Part (a) follows immediately from the remark following Definition 1.1. See also [9, Proposition (19.3.5)]. By [11, Theorem 5],

$$
H^{2}\left(\bigotimes_{i \in I} A_{i}, M\right)^{s} \cong \prod_{i \in I} H^{2}\left(A_{i}, M\right)^{s}
$$

and so we have part (b).

Consider (c). By Lemma 3.1 applied to the complex $C_{*}(A / k)^{s}$ with $m=1$ and $n=2$, we have that $H^{2}(A, M)^{s} \cong \operatorname{Hom}_{A}\left(H_{2}(A / k)^{s}, M\right)$ since $\Omega_{A / k} \cong H_{1}(A / k)^{s}$ is projective. Since $H_{2}(A / k)^{s} \cong$ inj $\lim H_{2}\left(A_{i} / k\right)^{s}$, it will suffice to show that $H_{2}\left(A_{i} / k\right)^{s}$ $=0$. By the same reasoning as above, we have that $H^{2}\left(A_{i}, N\right)^{s} \cong \operatorname{Hom}_{A_{i}}\left(H_{2}\left(A_{i} / k\right)^{s}, N\right)$ since $\Omega_{A_{i} / k}$ is a projective $A_{i}$-module (Corollary 2.4). Thus since $A_{i}$ is a formally smooth $k$-algebra $H_{2}\left(A_{i} / k\right)^{s}=0$.

The following theorem is a generalization of [11, Corollary 23] and the footnote of [11] added in proof.

THEOREM 5.3. Let $A$ be a $k$-algebra and let $A=B / I$ where $B$ is a formally smooth $k$-algebra. The following conditions are equivalent:

(i) $A$ is a formally smooth $k$-algebra.

(ii) $H^{2}\left(A, I / I^{2}\right)^{s}=0$.

(iii) The $k$-algebra extension $0 \rightarrow I / I^{2} \rightarrow B / I^{2} \rightarrow A \rightarrow 0$ is equivalent to the trivial k-algebra extension of $A$ by $I / I^{2}$. 
If $I / I^{2}$ is a finitely generated $A$-module, the above conditions are equivalent to:

(iv) $H^{2}(A, M)^{s}=0$ for all finitely generated $A$-modules $M$.

Proof. Clearly (i) implies (ii), (ii) implies (iii), and (i) implies (iv). Since (iv) implies (ii) whenever $I / I^{2}$ is finitely generated, it will suffice to show that (iii) implies (i).

Since $0 \rightarrow I / I^{2} \rightarrow B / I^{2} \stackrel{\gamma}{\longrightarrow} A \rightarrow 0$ is equivalent to the trivial $k$-algebra extension of $A$ by $I / I^{2}$, there is a $k$-algebra map $\rho: A \rightarrow B / I^{2}$ such that $\gamma \rho=1$. Hence $1-\rho \gamma$ maps $B / I^{2}$ into $\operatorname{ker}(\gamma)=I / I^{2}$ since $\gamma(1-\rho \gamma)=\gamma-\gamma \rho \gamma=0$. Now $I / I^{2}$ has square zero and so for all $a, b \in B / I^{2}$

$$
\begin{aligned}
(1-\rho \gamma)(a)(1-\rho \gamma)(b) & =0 \\
& =a b-a \rho \gamma(b)-b \rho \gamma(a)+\rho \gamma(a) \rho \gamma(b) .
\end{aligned}
$$

Hence $a b-\rho \gamma(a) \rho \gamma(b)=a b-a \rho \gamma(b)+a b-b \rho \gamma(a)$. Thus $D=1-\rho \gamma \in \operatorname{Der}_{k}\left(B / I^{2}, I / I^{2}\right)$. Note also that $\left.D\right|_{I / I^{2}}=1$.

Let $M$ be an $A$-module. We obtain the exact sequence

$$
H^{1}(B, M)^{s} \stackrel{\omega}{\longrightarrow} \operatorname{Hom}_{B}(I, M) \longrightarrow H^{2}(A, M)^{s} \longrightarrow 0
$$

by using exact sequence (1) of $\S 3$. $\omega$ is defined by $\omega(d)=\left.d\right|_{I}$. Since $I M=0$, $\operatorname{Hom}_{B}(I, M) \cong \operatorname{Hom}_{B / I}\left(I / I^{2}, M\right)$ and $H^{1}(B, M)^{s}=\operatorname{Der}_{k}(B, M) \cong \operatorname{Der}_{k}\left(B / I^{2}, M\right)$ $=H^{1}\left(B / I^{2}, M\right)^{s}$. Hence if $f \in \operatorname{Hom}_{B / I}\left(I / I^{2}, M\right)$, we have that $\omega(f D)=f$ since $\left.D\right|_{I / I^{2}}=1$ where $D$ is the derivation defined above. Thus $\omega$ is onto and so $H^{2}(A, M)^{s}$ $=0$.

Definition 5.4. Let $A$ be a $k$-algebra. We say that $A$ is a geometric $k$-algebra if $A$ is a localization of a finitely generated $k$-algebra.

Theorem 5.5. Let $A$ be a k-algebra such that $H^{3}(A, M)^{s}=0$ whenever $M$ is a submodule of a free $A$-module. The following conditions are equivalent:

(i) $H^{2}(A, M)^{s}=0$ for all finitely generated $A$-modules $M$.

(ii) $H^{2}(A, A)^{s}=0$.

(iii) $A$ is a rigid $k$-algebra in the commutative deformation theory. If in addition, $A$ is a geometric $k$-algebra, then the above conditions are equivalent to:

(iv) $A$ is a formally smooth k-algebra.

Proof. Since $H^{3}(A, A)^{s}=0$, (ii) and (iii) are equivalent. By Theorem 5.3, (i) and (iv) are equivalent since we may choose $I$ to be a finitely generated ideal in the presentation of $A$ as $B / I$. It will suffice to show that (ii) implies (i).

Let $N$ be a finitely generated $A$-module and let $0 \rightarrow K \rightarrow F \rightarrow N \rightarrow 0$ be an exact sequence where $F$ is a finitely generated free module. This sequence induces the exact sequence

$$
H^{2}(A, F)^{s} \rightarrow H^{2}(A, N)^{s} \rightarrow H^{3}(A, K)^{s}=0
$$

by [11, Theorem 1]. But since $F=\bigoplus_{1 \leqq i \leqq n} A_{i}, A_{i}=A$ for each $i$, we have that $H^{2}(A, F)^{s} \cong \oplus_{1 \leqq i \leqq n} H^{2}\left(A, A_{i}\right)^{s}=0$. 
We refer the reader to [20] for the definition and properties of regular local rings. We need the following theorem which is due to Harrison [11, Theorem 19, Theorem 22] but has a weaker hypothesis.

TheOREM 5.6 (HARrison). Let $A$ be a local $k$-algebra such that $A / \mathfrak{M}$ is a separable extension of $k$ where $\mathfrak{M}$ is the maximal ideal of $A$. The following conditions are equivalent:

(i) $H^{2}(A, N)^{s}=0$ for all $A / \mathfrak{M}$-modules $N$.

(ii) $H^{2}(A, A / \mathfrak{M})^{s}=0$.

(iii) $A$ is a regular local ring.

If $A$ is a geometric local $k$-algebra, then the above are equitalent to the following conditions:

(iv) $A$ is a formally smooth $k$-algebra.

(v) $H^{2}(A, N)^{s}=0$ for all finitely generated $A$-modules $N$.

Proof. We may remove Harrison's restriction that $k$ be perfect by using the fact that a separable extension of $k$ is a formally smooth $k$-algebra [8, Theorem 3]. By Theorem 5.3, (v) implies (iv).

Remark 5.7. Let $A$ be a $k$-algebra and let $k_{0}$ be a subfield of $k$ with $k$ a separable extension of $k_{0}$. By utilizing the exact sequence of [9, Corollary (20.2.3)] and [8, Theorem 3], we may easily show that if $A$ is a formally smooth $k$-algebra, then $A$ is a formally smooth $k_{0}$-algebra. In particular, let $A$ be a formally smooth noetherian local $k$-algebra. Since every extension of the prime field $k_{0}$ of $k$ is a separable extension, it follows immediately that $A$ is a regular local ring.

COROLlaRy 5.8. Let $A$ be a k-algebra with a noetherian total quotient ring $S^{-1} A$. If $S^{-1} A$ is a formally smooth algebra over some subfield of $k$, then $A$ is reduced (=no nonzero nilpotent elements). Hence a noetherian formally smooth $k$-algebra is reduced.

Proof. The final assertion follows from the first assertion since the map $A \rightarrow S^{-1} A$ induces an isomorphism $H^{2}\left(S^{-1} A, M\right)^{s} \cong H^{2}(A, M)^{s}$ where $M$ is an $S^{-1} A$-module [11, Theorem 16].

Since $A$ is contained in $S^{-1} A$, it will suffice to show that $S^{-1} A$ is reduced. By Remark 5.7, each localization of $S^{-1} A$ at a prime ideal is a regular local ring. But the nilradical of a ring is zero if and only if the nilradical of each of its localizations is zero. Hence $S^{-1} A$ is reduced.

Proposition 5.9. Let $A$ be an artinian $k$-algebra. Then $A$ is a formally smooth $k$-algebra if and only if $A$ is a product of separable extension fields of $k$.

Proof. If $A$ is a product of separable extension fields of $k$, then $A$ is a formally smooth $k$-algebra by Proposition 5.2(a) since a separable extension of $k$ is a formally smooth $k$-algebra [8, Theorem 3]. 
Conversely, assume that $A$ is a formally smooth $k$-algebra. Since $A \cong \prod_{\mathfrak{M}} A_{\mathfrak{M}}$ with the product over the set of maximal ideals $\mathfrak{M}$ of $A$, we may assume that $A$ is a local algebra by the remark following Definition 1.1. But by Remark 5.7, $A$ is a regular artinian local $k$-algebra and so $A$ is a field. Hence $A$ must be a separable extension of $k$.

Lemma 5.10. Let $A$ be a k-algebra with an artinian formally smooth total quotient ring. Suppose that $H^{3}(A, N)^{s}=0$ whenever $N$ is a submodule of a free A-module. If $M$ is any A-module, then

$$
\mu_{1}: \operatorname{Ext}_{A}^{1}\left(\Omega_{A / k}, M\right) \rightarrow H^{2}(A, M)^{s}
$$

is an isomorphism where $\mu_{1}$ is the map of Proposition 2.3.

Proof. Let $0 \rightarrow K \rightarrow F \rightarrow M \rightarrow 0$ be an exact sequence of $A$-modules with $F$ free. Thus $\operatorname{Ext}_{A}^{2}\left(\Omega_{A / k}, K\right)=0$ by Proposition 2.5. Setting $\Omega=\Omega_{A / k}$, we obtain the following commutative diagram with exact rows.

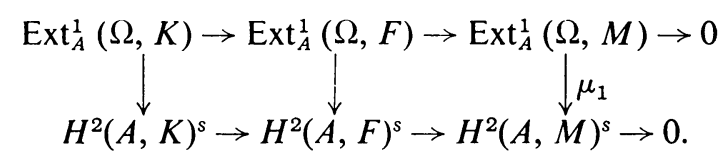

The first two vertical arrows are isomorphisms by Proposition 2.5. Hence $\mu_{1}$ is an isomorphism.

THEOREM 5.11. Let $A$ be a k-algebra with an artinian formally smooth total quotient ring. Suppose that $H^{3}(A, N)^{s}=0$ whenever $N$ is a submodule of a free $A$ module. The following conditions are equicalent:

(i) $A$ is a formally smooth $k$-algebra.

(ii) $\Omega_{A / k}$ is a projective A-module.

If in addition $A$ is a geometric $k$-algebra, then the above are equivalent to the following conditions:

(iii) $H^{2}(A, M)^{s}=0$ for all finitely generated $A$-modules $M$.

(iv) $H^{2}(A, A)^{s}=0$.

(v) $A$ is a rigid $k$-algebra in the commutative deformation theory.

Proof. By Lemma 5.10, (ii) is equivalent to (i). Conditions (i), (iii), (iv), and (v) are equivalent by Theorem 5.5 .

6. Complete intersections and a conjecture of Gerstenhaber.

Definition 6.1. Let $A$ be a ring and $M$ an $A$-module. We say that an element $f \in A$ is $M$-regular if $f$ is not a zero-divisor in $M$. A sequence $\left(f_{i}\right)_{1 \leqq i \leqq n}$ of elements of $A$ is an $M$-regular sequence if for each $i=1, \ldots, n, f_{i}$ is $M_{i-1}$-regular where $M_{0}=M$ and $M_{i-1}=M /\left(f_{1}, \ldots, f_{i-1}\right) M$ for $i>1$.

THEOREM 6.2. Let $B$ be a $k$-algebra and let $I$ be an ideal of $B$ which is generated by a $B$-regular sequence. If $M$ is a B/I-module, then the map

$$
p_{n}: H^{n}(B / I, M)^{s} \rightarrow H^{n}(B, M)^{s}
$$


induced by the projection $B \rightarrow B / I$ is an epimorphism for $n=2$ and a monomorphism for $n=3$.

Proof. Let $\left(f_{i}\right)_{1 \leqq i \leqq n}$ be a $B$-regular sequence which generates $I$. Let $B_{i}=B /\left(f_{1}, \ldots, f_{i}\right)$; thus $B_{0}=B$ and $B_{n}=B / I$. Since $f_{i}^{\prime}$, the image of $f_{i}$ in $B_{i-1}$, is not a zero-divisor in $B_{i-1}, f_{i}^{\prime} B_{i-1}$ is a free ideal. Hence $\operatorname{Ext}_{B_{i-1}}^{1}\left(f_{i}^{\prime} B_{i-1}, M\right)=0$ and so $C^{1}\left(B_{i-1}, f_{i}^{\prime} B_{i-1}, M\right)=0$ using the notation of exact sequence (1), $\$ 3$. Thus we have a sequence of epimorphisms

$$
H^{2}\left(B_{n}, M\right)^{s} \rightarrow H^{2}\left(B_{n-1}, M\right)^{s} \rightarrow \cdots \rightarrow H^{2}\left(B_{0}, M\right)^{s}
$$

and the composite is the required epimorphism. We use a similar argument for the final assertion.

Definition 6.3. Let $A$ be a $k$-algebra. We say that $A$ is a geometric complete intersection if $A$ is a localization of a $k$-algebra of the form $k\left[X_{1}, \ldots, X_{n}\right] /\left(f_{1}, \ldots, f_{m}\right)$ where $\left(f_{i}\right)_{1 \leqq i \leqq m}$ is a $k\left[X_{1}, \ldots, X_{n}\right]$-regular sequence.

We recall that a noetherian local ring $A$ is said to be a local complete intersection if there is a regular local ring $B$ such that $A \cong B / I$ where $I$ is an ideal such that the minimal number of generators of $I$ is equal to $\operatorname{dim}(B)-\operatorname{dim}(A)$ (Krull dimension). We may choose a set of generators for $I$ which form a $B$-regular sequence [12], [21]. However, note that we do not require that a geometric complete intersection be a local ring.

Corollary 6.4. Let $A$ be a k-algebra and $M$ an $A$-module.

(a) If $A$ is a geometric complete intersection, then $H^{3}(A, M)^{s}=0$.

(b) If $A$ is a semisimple k-algebra, then $H^{3}(A, M)^{s}=0$.

Proof. Part (a) follows immediately from Theorem 3.3 and Theorem 6.2.

Consider part (b). By the remark following Definition 1.1, we may assume that $A$ is an extension field of $k$. Now since $A$ is a field, $H^{3}(A, M)^{s} \cong \operatorname{Hom}_{A}\left(H_{3}(A / k)^{s}, M\right)$. Since $A \cong \operatorname{inj} \lim A_{i}$ where the direct limit is over the set of subfields of $A$ which are finitely generated over $k$, we have that $H_{3}(A / k)^{s} \cong \operatorname{inj} \lim H_{3}\left(A_{i} / k\right)^{s}$. Hence we may assume that $A$ is a finitely generated extension field of $k$. But then $A$ is a regular geometric complete intersection and so $H_{3}(A / k)^{s}=0$ by part (a).

Note that Corollary 6.4(a) generalizes [11, footnote 4]. The following theorem answers a conjecture of Gerstenhaber [8, p. 80].

THEOREM 6.5. Let $A$ be a semisimple k-algebra. The following conditions are equivalent:

(i) $A$ is a formally smooth k-algebra.

(ii) $H^{2}(A, A)^{s}=0$.

(iii) $A$ is a rigid k-algebra in the commutative deformation theory.

(iv) $A$ is a product of separable extension fields of $k$.

Proof. Conditions (i) and (iv) are equivalent by Proposition 5.9 and (ii) and (iii) are equivalent since $H^{3}(A, A)^{s}=0$. Since $A$ is semisimple, we have that $H^{2}(A, M)^{s}$ 
$\cong \operatorname{Hom}_{A}\left(H_{2}(A / k)^{s}, M\right)$. Thus if $H^{2}(A, A)^{s}=0$, we have that $H_{2}(A / k)^{s}=0$ and so (ii) implies (i).

If $A$ is an $L$-algebra and if $k$ is a subfield of $L$, we shall let $H_{k}^{n}(A, M)^{s}$ denote the $n$th commutative cohomology group of $A$ considered as a $k$-algebra.

Proposition 6.6. Let $A$ be an L-algebra which is a geometric complete intersection and let $M$ be an $A$-module. If $k$ is any subfield of $L$, then $H_{k}^{3}(A, M)^{s}=0$.

Proof. By Corollary 6.4(b), Theorem 4.1, and Theorem 3.3, the 3rd cohomology group of the $k$-algebra $S^{-1} L\left[X_{1}, \ldots, X_{n}\right]$ vanishes. Hence $H_{k}^{3}(A, M)^{s}=0$ by applying Theorem 6.2.

COROLLARY 6.7. Let $A$ be an L-algebra which is a geometric complete intersection and let $k$ be a subfield of $L$. If the total quotient ring of $A$ is an artinian formally smooth $k$-algebra, then the projective dimension of $\Omega_{A / k}$ is $\leqq 1$.

Proof. Apply Proposition 6.6, Proposition 5.9, and Corollary 2.6(a).

THEOREM 6.8. Let $A$ be an L-algebra which is a geometric complete intersection and let $k$ be any subfield of $L$.

(a) The following conditions are equiralent:

(i) $A$ is a formally smooth k-algebra.

(ii) $H_{k}^{2}(A, M)^{s}=0$ for all finitely generated $A$-modules $M$.

(iii) $H_{k}^{2}(A, A)^{s}=0$.

(iv) $A$ is a rigid k-algebra in the commutative deformation theory.

(b) If $A$ is also a local ring with maximal ideal $\mathfrak{M}$ and if $A / \mathfrak{M}$ is a separable extension of $k$, then the conditions of (a) are equivalent to:

(v) $A$ is a regular local ring.

(c) If the total quotient ring of $A$ is an artinian formally smooth $k$-algebra, then the conditions of (a) are equivalent to:

(vi) $\Omega_{A / k}$ is a projective A-module.

Proof. The equivalence of the conditions of (a) follows immediately from Proposition 6.6 and the reasoning of Theorem 5.5. Part (b) is Theorem 5.6. Part (c) is an application of Theorem 5.11.

7. Group algebras. Let $G$ be a group and $A$ a ring. The group algebra of $G$ over $A, A[G]$, is the free $A$-module with basis $\left(x_{g}\right)_{g \in G}$ where the multiplication is defined by $x_{g} x_{h}=x_{g h}$. We shall be concerned only with abelian groups $G$ so that the algebra $A[G]$ will be commutative. If $G$ and $G^{\prime}$ are abelian groups, we have that $A\left[G \oplus G^{\prime}\right] \cong A[G] \otimes_{A} A\left[G^{\prime}\right]$ and, if $k$ is a subring of $A, A[G] \cong A \otimes_{k} k[G]$.

LEMMA 7.1. Let $G$ be an abelian group which is a direct sum of cyclic groups and let $A$ be a k-algebra. If $H^{3}(A, M)^{s}=0$ for all $A$-modules $M$, then $H^{3}(A[G], N)^{s}=0$ for all $A[G]$-modules $N$. 
Proof. Since the 3rd cohomology group of a tensor product of $k$-algebras is isomorphic to the product of the 3rd cohomology groups of the algebras [11, Theorem 5], it will suffice to consider $H^{3}(k[G], M)^{s}$. By hypothesis, $G=\oplus_{i \in I} C_{i}$ where each $C_{i}$ is cyclic. Since $k[G] \cong \bigotimes_{i \in I} k\left[C_{\imath}\right]$, we may assume that $G=\boldsymbol{Z}$ or $G=\boldsymbol{Z} / n \boldsymbol{Z}$ again using [11, Theorem 5]. But $k[Z] \cong k[X, Y] /(X Y-1)$ and $k[\boldsymbol{Z} / n \boldsymbol{Z}]$ $\cong k[X] /\left(X^{n}-1\right)$ and each of these algebras is a geometric complete intersection and so we may apply Corollary 6.4(a).

THEOREM 7.2. Let $G$ be an abelian group which is a direct sum of cyclic groups and let $A$ be a formally smooth k-algebra.

(a) If char $(k)=0$ or if $\operatorname{char}(k)=p \neq 0$ and no element of $G$ has order $p$, then $A[G]$ is a formally smooth $k$-algebra.

(b) If char $(k)=p \neq 0$ and $A$ is noetherian, then $A[G]$ is a formally smooth $k$ algebra if and only if $G$ has no element of order $p$.

Proof. By the argument used in the proof of Lemma 7.1, it will suffice to prove part (a) for $A=k$ and for $G=Z$ and $G=Z / n Z$. Under either of the hypotheses of part (a), $X^{n}-1$ is a separable polynomial and so $k[Z / n Z] \cong k[X] /\left(X^{n}-1\right)$ is a product of separable extensions of $k$. Hence by Proposition $9.10, k[Z / n Z]$ is a formally smooth $k$-algebra. Now

$$
k[Z] \cong k[X, Y] /(X Y-1) \cong k[X, 1 / X]
$$

is a localization of a polynomial $k$-algebra and so $k[Z]$ is a formally smooth $k$-algebra [11, Theorem 15].

Conversely, suppose that $A[G]$ is a formally smooth $k$-algebra with char $(k)$ $=p \neq 0$ and $A$ a noetherian $k$-algebra. Assume that $G$ has a direct summand of the form $\boldsymbol{Z} / n \boldsymbol{Z}$ with $n=p m$. The projection $G \rightarrow \boldsymbol{Z} / n \boldsymbol{Z}$ induces a map $p: A[G] \rightarrow$ $A[Z / n Z]$ and the composite

$$
A[\boldsymbol{Z} / n \boldsymbol{Z}] \stackrel{i}{\longrightarrow} A[G] \stackrel{p}{\longrightarrow} A[\boldsymbol{Z} / n \boldsymbol{Z}]
$$

is the identity where $i$ is induced by the inclusion $Z / n Z \rightarrow G$. Let $M$ be an $A[Z / n Z]$ module. Considering $M$ as an $A[G]$-module via $p$, we have that $H^{2}(A[G], M)^{s}=0$. If $G=\boldsymbol{Z} / n \boldsymbol{Z} \oplus G^{\prime}$, then

$$
H^{2}(A[G], M)^{s} \cong H^{2}(A[\boldsymbol{Z} / n Z], M)^{s} \oplus H^{2}\left(k\left[G^{\prime}\right], M\right)^{s}=0
$$

by [11, Theorem 5]. Since $p i=1, A[Z / n Z]$ is a formally smooth $k$-algebra. Thus $A[\boldsymbol{Z} / n \boldsymbol{Z}]$ is a reduced algebra by Corollary 5.8. Write $A[\boldsymbol{Z} / n Z]=A[X] /\left(X^{n}-1\right)$ $=A[x]$ where $x=X+\left(X^{n}-1\right)$. Since $n=p m, x^{m}-1$ is nilpotent of order $p$. But $x^{m}-1 \neq 0$ and so $n \neq p m$.

COROLLARY 7.3. Let $G$ be a finitely generated abelian group with torsion subgroup $T$. Let $A$ be a formally smooth $k$-algebra which is either semisimple or a geometric complete intersection. If $\operatorname{char}(k)=p \neq 0$, then the following conditions are equivalent:

(i) $A[G]$ is a formally smooth k-algebra. 
(ii) $H^{2}(A[G], A[G])^{s}=0$.

(iii) $A[G]$ is a rigid k-algebra in the commutative deformation theory.

(iv) $p$ does not dicide the order of $T$.

\section{BIBLIOGRAPHY}

1. N. Bourbaki, Algèbre, Actualités Sci. Indust., No. 1236, Hermann, Paris, 1962.

2. - Algèbre commutative, Actualités Sci. Indust., No. 1290, Hermann, Paris, 1961.

3. - Algèbre commutative, Actualités Sci. Indust., No. 1293, Hermann, Paris, 1961.

4. H. Cartan and S. Eilenberg, Homological algebra, Princeton Univ. Press, Princeton, N. J., 1956.

5. P. Cartier, Dérivations dans les corps, Séminaire H. Cartan et C. Chevalley, 8e année, 1955-1956, exposé 13.

6. M. Gerstenhaber, On the cohomology structure of an associative ring, Ann. of Math. (2) 78 (1963), 267-288.

7. - On the construction of division rings by the deformation of fields, Proc. Nat. Acad. Sci. U. S. A. 55 (1966), 690-692.

8. - On the deformation of rings and algebras, Ann. of Math. (2) 79 (1964), 59-103.

9. A. Grothendieck, Éléments de géométrie algébrique. IV. Étude locale des schémas et des morphismes de schémas. I, Inst. Hautes Études Sci. Publ. Math. No. 20 (1964).

10. - Sur quelques points d'algèbre homologique, Tôhoku Math. J. 9 (1957), 119-221.

11. D. Harrison, Commutative algebras and cohomology, Trans. Amer. Math. Soc. 104 (1962), 191-204.

12. R. Hartshorne, Complete intersections and connectedness, Amer. J. Math. 84 (1962), 497-508.

13. G. Hochschild, On the cohomology theory for associative algebras, Ann. of Math. (2) 47 (1946), 568-579.

14. G. Hochschild, B. Kostant and A. Rosenberg, Differential forms on regular affine algebras, Trans. Amer. Math. Soc. 102 (1962), 383-408.

15. N. Jacobson, Lectures in abstract algebra, Vol. III, Van Nostrand, Princeton, N. J., 1964.

16. S. MacLane, Homology, Springer-Verlag, Berlin, 1963.

17. A. Nijenhuis, Graded Lie algebras and their applications, Universiteit van Amsterdam, Mimeographed Notes, 1964.

18. A. Rosenberg and D. Zelinsky, Cohomology of infinite algebras, Trans. Amer. Math. Soc. 82 (1956), 85-98.

19. P. Samuel, Méthodes d'algèbre abstraite en géométrie algébrique, Ergebnisse der Math., Heft 4, Springer-Verlag, Berlin, 1955.

20. J.-P. Serre, Algèbre locale-multiplicités, Lecture Notes in Mathematics 11, SpringerVerlag, Berlin, 1965.

21. S. Suzuki, On torsion in the module of differentials of a locality which is a complete intersection, J. Math. Kyoto Univ. 4 (1964-1965), 471-475.

22. O. Zariski and P. Samuel, Commutative algebra, Vol. II, Van Nostrand, Princeton, N. J., 1960.

\section{CORNELL UNIVERSITY, ITHACA, New York}

